\title{
Effects of mobile vacancies on the dynamics of ordering and phase separation in nonconserved multicomponent systems
}

\section{Gilhøj, Henriette; Jeppesen, Claus; Mouritsen, Ole G.}

Published in:

Physical Review E. Statistical, Nonlinear, and Soft Matter Physics

Link to article, DOI:

10.1103/PhysRevE.52.1465

Publication date:

1995

Document Version

Publisher's PDF, also known as Version of record

Link back to DTU Orbit

Citation (APA):

Gilhøj, H., Jeppesen, C., \& Mouritsen, O. G. (1995). Effects of mobile vacancies on the dynamics of ordering and phase separation in nonconserved multicomponent systems. Physical Review E. Statistical, Nonlinear, and Soft Matter Physics, 52(2), 1465-1475. https://doi.org/10.1103/PhysRevE.52.1465

\section{General rights}

Copyright and moral rights for the publications made accessible in the public portal are retained by the authors and/or other copyright owners and it is a condition of accessing publications that users recognise and abide by the legal requirements associated with these rights.

- Users may download and print one copy of any publication from the public portal for the purpose of private study or research.

- You may not further distribute the material or use it for any profit-making activity or commercial gain

- You may freely distribute the URL identifying the publication in the public portal 


\title{
Effects of mobile vacancies on the dynamics of ordering and phase separation in nonconserved multicomponent systems
}

\author{
Henriette Gilhøj, ${ }^{1}$ Claus Jeppesen, ${ }^{2}$ and Ole G. Mouritsen ${ }^{1}$ \\ ${ }^{1}$ Department of Physical Chemistry, The Technical University of Denmark, Building 206, DK-2800 Lyngby, Denmark \\ ${ }^{2}$ Materials Research Laboratory, University of California, Santa Barbara, California 93106
}

(Received 25 January 1995)

\begin{abstract}
The effects of mobile vacancies on the dynamics of ordering processes and phase separation in multicomponent systems are studied via Monte Carlo simulations of a two-dimensional seven-state ferromagnetic Potts model with varying degrees of site dilution. The model displays phase equilibria corresponding to a dilute Potts-disordered (fluid) phase and a dilute Potts-ordered phase (solid), as well as a broad region of coexistence between the fluid and the solid phase. Temperature quenches into the dilute Potts-ordered phase as well as into the phase-separated region are considered under the condition of conserved vacancy density and nonconserved Potts order. The dynamics of ordering and phase separation is found to follow algebraic growth laws with exponent values that depend on the phase to which the quench is performed. Strong transient effects are observed in the dilute Potts-ordered phase, which are shown to result from an accumulation of vacancies at the domain boundaries. These transient effects are accompanied by an overshooting effect in the local order of the growing domains.
\end{abstract}

PACS number(s): 05.70.Ln, 64.60.My, 64.60.Cn, 75.40.Mg

\section{INTRODUCTION}

Systems undergoing far-from-equilibrium ordering processes after being quenched in temperature are known to display a remarkable degree of universality in the dynamic behavior of the ordering domains [1-5]. Specifically, it has been found for a number of ideal and pure systems that the average linear size $R(t)$ of the growing domains at late times obeys algebraic growth laws

$$
R(t) \sim A t^{n},
$$

with values of the growth exponent $n$ that characterize large universality classes [6] that are mainly determined by the conservation laws in effect. Moreover, it is found that at late times only a single time-dependent length scale is needed to characterize the essential time dependence of the problem, and the ordering process exhibits dynamical scaling [1].

For impure and imperfect systems, the situation is more complex and much more controversial due to the randomness that these complications introduce into the problem [7]. Several types of imperfections have been studied, including site impurities [8-13], vacancies [14-29], random couplings [30,31], random fields [32-43], as well as second-phase particles [44]. When site impurities or site dilution (vacancies) are involved in the ordering process, two major circumstances seem to be important when classifying the ordering dynamics. First, it is relevant whether the impurities (or vacancies) are mobile (annealed) or not (quenched). If they are quenched they tend to pin the domain interfaces, leading to activated processes and at late times to a logarithmic growth behavior, $R(t) \sim(\ln t)^{\phi}$, where $\phi$ is some exponent [12]. If the impurities are annealed, their diffusive dynamics cou- ples to the motion of the domain interfaces [45] and the ordering dynamics now critically depends on the nature of the final equilibrium state of the system, which may be different from that of a pure system. In the case where the equilibrium state has the same symmetry as that of the pure system no changes in the essential time dependence of $R(t)$ is to be expected, although the amplitude $A$ in Eq. (1) may be drastically changed in the presence of mobile impurities that couple to the interface motion. Mobile impurities can, however, also lead to changes in the phase equilibria in a way that depends on the details of the interaction between the pure-system components and the impurities as well as the mutual interactions between the impurities. If the quench takes the system into regions of phase separation or phases with new symmetries, the ordering dynamics may be significantly altered.

For impurities that have no specific mutual interactions among themselves, and hence display no separate cooperativity, a fairly simple change in the phase equilibria arises in the case where the impurities are not interfacially active. This is the case for inert impurities and vacancies where the entropy of mixing may introduce phase coexistence between the phases of the pure-system component. Only under very special circumstances can the impurity induce phases of new symmetry. Examples include stoichiometric complexes with vacancy superstructures in antiferromagnets with competing interactions [21] and rotationally inert impurities in orientationally ordered structures [e.g., $\left(\mathbf{N}_{2}+\mathbf{A r}\right)$-graphite [15]]. If, however, the impurities are interfacially active, i.e., they tend specifically to lower the interfacial tension between ordered domains or phases, new phases may arise with different symmetries from that of the pure system. Wellknown examples are phases with microphase separation, 
e.g., in block copolymer mixtures or microemulsions like ternary oil-water-surfactant systems [46-49].

In order to gain information about the influence on ordering dynamics of mobile vacancies and in particular on the relevance of the phase to which a thermal quench is performed we have conducted a numerical study using a very simple statistical mechanical model that in the presence of vacancies has a simple phase diagram including both one-phase regions as well as a phase-separated region. The model is the two-dimensional seven-state ferromagnetic Potts model with annealed site dilution. The seven-state ferromagnetic Potts model in two dimensions is known to display a strong first-order phase transition from a Potts-ordered (solid) phase to a Potts-disordered (fluid) phase [50]. When vacancies are introduced, simple freezing-point depression results and a region of coexistence between the two phases is introduced. Hence this model is a good and simple candidate for a study of nonequilibrium ordering dynamics and how it may depend on diffusing vacancies and the nature of the phase to which the quench is performed. Since the Potts model has a degenerate ground state (in the case of the sevenstate ferromagnetic Potts model there exist seven thermodynamically equivalent states of different Potts order below the phase transition temperature), two types of ordering phenomena may occur subsequent to a quench. In both cases, the Potts spins order in ferromagnetic Pottsordered domains (with some dilution). Depending on temperature and vacancy concentration, a phaseseparation process may simultaneously take place between Potts-ordered domains and the Potts-disordered phase. Hence, the ordering processes involve ordering of the Potts spins as well as redistribution of the vacancies. Therefore, two ordering fields have to be taken into account, the Potts order parameter and the vacancy concentration. In the present paper we shall impose a global conservation law for the vacancy concentration whereas the Potts order is kept as a nonconserved quantity. These conservation laws shall turn out to be relevant for classifying the growth behavior.

In Sec. II we define the dilute Potts model used in our study and describe the Monte Carlo computer-simulation techniques used to derive the phase equilibria as well as the nonequilibrium ordering dynamics. This section also includes a short subsection on a particular numerical algorithm we have developed in order to make it computationally feasible to simulate the time evolution of interfaces in inhomogeneous systems. The main results of the study are reported in Sec. III, which describes the phase diagram, the morphology of the domain patterns, and the associated interfacial network, the growth laws, as well as the growth exponents. Furthermore, we present data for local order parameters that show that the nonequilibrium ordered domains have a structure and composition significantly different from that of the equilibrium phases. We describe this phenomenon as a kind of overshooting effect and discuss in Sec. IV the possible generic occurrence of overshooting effects in nonequilibrium ordering dynamics as due to a subtle competition between nonequilibrium internal energy and nonequilibrium entropy.

\section{MODEL AND SIMULATION METHOD}

\section{A. The dilute Potts model}

The model we have used for the present study is a version of the $q=7$ state Potts model defined by the Hamiltonian

$$
\begin{array}{r}
H=-J \sum_{\langle i, j\rangle}\left(\delta_{\sigma_{i}, \sigma_{j}}-\delta_{\sigma_{i}, q+1} \delta_{\sigma_{j}, q+1}\right), \\
\sigma_{i}=1,2, \ldots, q+1,
\end{array}
$$

with $J>0$ corresponding to ferromagnetic interactions. State $q+1$ is interpreted as a site vacancy. The (global) vacancy concentration is determined given by

$$
\rho=\left\langle N^{-1} \sum_{i} \delta_{\sigma_{i}, q+1}\right\rangle,
$$

where the angular brackets denote an ensemble average. The summation in Eq. (2) is over nearest-neighbor sites of a finite triangular lattice with periodic boundary conditions. We have studied a series of lattice sizes, $N=L \times L$, with $L=50,200,500,1000$. Most of the results reported below correspond to $L=200$.

The pure two-dimensional $q$-state Potts model has a first-order phase transition for $q>3$. For $q=7$, the transition is strongly first order and the transition temperature is known from exact calculations to be $k_{B} T_{c} / J=2 / \ln 3 \simeq 1.8205$ [50]. Below $T_{c}$, the model exhibits a Potts-ordered (PO) phase and above $T_{c}$, the model has a Potts-disordered (PD) phase. When the annealed site dilution is introduced, a region of coexistence between the two phases (PO-DO) is introduced. The precise phase diagram is described in Sec. III A (cf. Fig. 1).

\section{B. Model dynamics}

The Potts model in Eq. (2) has no natural dynamics and stochastic dynamics therefore has to be invoked in order to associate dynamical behavior with the model, both for providing equilibrium properties via the simulations and for deriving the nonequilibrium ordering dynamics.

In all simulations, except for those used to derive the equilibrium phase diagram, the vacancy concentration $\rho$ is kept fixed and is therefore a conserved field. The other relevant field is the (global) Potts order parameter,

$$
\Psi=\left\langle\frac{q N^{-1} \max _{\sigma}\left(\sum_{i} \delta_{\sigma, \sigma_{i}}\right)-1}{q-1}\right\rangle,
$$

which we keep as a nonconserved quantity.

The conservation of $\rho$ is assumed by only allowing vacancies to be involved in two-site Kawasaki exchanges, whereas the Potts spins are allowed to change state according to single-site Glauber dynamics, which does not conserve $\Psi$. These two types of dynamics have to be specified in relation to each other in order to define the detailed microscopic dynamics. We have chosen to consider the following two types of dynamics:

(A) Coupled dynamics. - Potts-spin changes are only allowed in connection with a Kawasaki exchange involv- 
ing a neighboring vacancy. The physics behind this mechanism is that the time evolution of the system proceeds via vacancy hopping, and the Potts spins are only allowed to relax in connection with a vacancy hop. The two processes are hence coupled and take place on the same time scale. Obviously, the pure Potts model limit cannot be studied by this type of dynamics.

(B) Uncoupled dynamics. - Single-site Potts-spin changes and vacancy exchange between neighboring sites involving a vacancy and a Potts spin are allowed to take place independently. This allows in principle for two different time scales. For simplicity we have taken the two independent processes to occur on the same time scale in order to facilitate comparison with (A).

In the Monte Carlo simulations the possible system updates are governed by the microscopic dynamics described above, and the acceptance procedure follows the usual Metropolis criterion, $\min \left(1, \exp \left(-\Delta E / k_{B} T\right)\right)$, where $\Delta E$ is the total internal energy associated with the update. In order to speed up the simulations, a particular fast updating algorithm has been developed as described in Sec. II E.

\section{Equilibrium and quenching simulations}

The calculation of the equilibrium phase diagram in the $T-\rho$ plane for the model in Eq. (2) proceeds via a grand-canonical simulation where a chemical-potential term, $-\mu \sum_{i} \delta_{\sigma_{i}, q+1}$, is added to the Hamiltonian. These calculations provide the phase boundaries with an accuracy that is sufficient to determine those state points, $(T, \rho)$, for the subsequent quench simulations, which are safely within a prescribed region of the phase diagram.

The quenching simulations are performed by preparing the system at a prescribed vacancy concentration in the PO phase, usually a completely random state corresponding to infinite temperature. The temperature is then suddenly changed to a temperature within either the PO or the PO-PD phase, and the microscopic dynamical evolution is governed by the principles described in Sec. II B. The time is hence naturally measured in units of Monte Carlo steps per site (MCS). In order to provide accurate ensemble averages for the measured time-dependent quantities, which in most cases are non-self-averaging [51], a large number of independent quenches, from 10 to 100 , have been performed for each value of temperature and vacancy concentration.

\section{Measures of time-dependent length scales}

The time evolution of the spatial ordering is followed by a number of different measures. First, a series of selected and representative microconfigurations are inspected qualitatively. Second, a measure of the average linear dimension, $R(t)$, of the growing domains is determined from the domain-area distribution function, $P(A, t)$, as

$$
R(t)=\left\langle\int_{A_{0}} A(T) P(A, t) d A\right\rangle^{1 / 2}
$$

where $A(t)$ is the area of an ordered Potts domain at time $t$. An ordered Potts domain is defined as a connected region of lattice sites that are occupied by Potts spins in the same state. Connectivity requires at least one nearest-neighbor bond. $A_{0}$ is a lower cutoff on the distribution function. The lower cutoff is chosen so as to eliminate effects due to small clusters of excitations, which should not be considered real domains. The choice of cutoff value (in the present case $A_{0}=4$ ) has no influence on the asymptotic behavior, but for finite-time simulations an appropriate choice of $A_{0}$ facilitates determination of the asymptotic behavior at an earlier time.

Furthermore we have monitored local aspects of the ordering and coarsening process by calculating two measures of local order. One is the local domain Potts order parameter

$$
\Psi_{\mathrm{loc}}(t)=\left\langle\frac{\overline{q N_{k}^{-1} \max _{\sigma}\left[\sum_{i=1}^{N_{k}} \delta_{\sigma, \sigma_{i}}\right]}-1}{q-1}\right\rangle
$$

and the other is the local concentration of vacancies within the ordered Potts domains

$$
\rho_{\mathrm{loc}}(t)=\left\langle N_{k}^{-1} \sum_{i=1}^{N_{k}} \delta_{\sigma_{i}}, \sigma .\right.
$$

In Eqs. (6) and (7) the bar refers to an average over all Potts-ordered domains and the sums are for each domain $k$ extended over the $N_{k}$ sites that make up the domain. In this averaging, only domains over a certain size, $N_{k} \geq 100$, are included in order to improve the statistical accuracy. The effects to be described in Sec. III D do not depend on this particular choice of smallest domain size. Furthermore, in the calculation of these local domain order parameters the same lower cutoff is introduced for the domain size as in Eq. (5) in order to eliminate effects from small clusters of correlated Potts spins or small vacancy precipitates. In the long-time limit, the two local order parameters tend towards the global, thermodynamic ordering fields, $\Psi_{\text {loc }}(t \rightarrow \infty)=\Psi(T)$ and $\rho_{\text {loc }}(t \rightarrow \infty)$ $=\rho(T)$.

\section{E. Fast updating algorithm}

The dynamics of ordering in far-from-equilibrium systems involves at intermediate and late times very heterogeneous states characterized by domains with a high degree of order (almost that of the equilibrium state) and a random network of interfaces corresponding to disorder. From the standpoint of stochastic Monte Carlo simulation it is therefore extremely inefficient to use updating algorithms that correspond to random or sequential updating of the lattice. The action is mostly related to the interfaces or those sites of the ordered domains where vacancies or broken Potts bonds occur. We have therefore developed a fast updating algorithm that focuses on those sites of the lattice that are susceptible to effective updates. This method was also employed in a previous study of ordering dynamics in conserved Potts models [52].

In the case of coupled dynamics (A), which is formally 
equivalent to ordinary two-site Kawasaki exchange [52], the algorithm is implemented in the following way. We maintain a list of links where one site at the edge of the link is occupied by a spin and the other by a vacancy. A link with this property is called a broken bond. We maintain a counter, $N_{\text {DIFF }}$, that keeps track of the number of broken bonds.

At every time step we choose a link randomly from the list and attempt an exchange between the site with the spin and the site with the vacancy. In the process we allow the spin to attempt a change of its value. We use the standard Metropolis criterion to decide whether or not to accept the combined move. The exchange, if accepted, will on the triangular lattice affect ten links which have to be either deleted from or added to the list of broken bonds. These changes may affect $N_{\text {DIFF }}$. Every time an attempt to update a broken bond has been performed we update a clock counting the number, $t_{\mathrm{MCBB}}$, of updated broken bonds per site according to $t_{\mathrm{MCBB}} \rightarrow t_{\mathrm{MCBB}}+1 / N$ where $N$ is the number of lattice sites.

This time scale for $t_{\mathrm{MCBB}}$ is obviously not the physical time scale associated with random activity on the lattice. Such a time scale would be simulated by a Metropolis algorithm where bonds are randomly selected, although in that case we might have to add the rule that time is not incremented if a bond with two vacancies is selected. At this point we run into the subtle problem of deciding whether a vacancy is considered a physical entity or not. In order to revert to the physical time scale we maintain yet another counter, $N_{\mathrm{PHYS}}=3 N-V$, where $V$ is the number of links with edges populated solely by vacancies. Every time a broken bond is selected for update we increment the physical time, $t_{\mathrm{MCS}}$, according to the rule

$$
t_{\mathrm{MCS}} \rightarrow t_{\mathrm{MCS}}+N_{\mathrm{PHYS}} /\left(N_{\mathrm{DIFF}} N\right)
$$

since on average we should have selected $N_{\text {PHYS }} / N_{\text {DIFF }}$ random bonds before we would have found a broken one. The factor of $N$ is a convention since $t_{\mathrm{MCS}}$ is defined as Monte Carlo steps per site.

With the relation in Eq. (8) we can check the validity of the time scale, $t_{\mathrm{MCS}}$, by noting that for low concentration of vacancies (so low that the vacancies do not aggre- gate), $t_{\mathrm{MCS}}$ and $t_{\mathrm{MCBB}}$ have to be proportional to each other, and the proportionality constant is the concentration of vacancies $\rho$.

This updating algorithm is very efficient since bonds selected for update are only those where an exchange leads to a physical change of the configuration. The price we pay for the efficiency is the extra overhead introduced with maintaining the list of broken bonds. Hence the algorithm becomes more economic during the late stages of the growth process where only few broken bonds remain. An interesting consequence of the algorithm is that the physical time, $t_{\mathrm{MCS}}$, via Eq. (8) becomes of a statistical nature. The precision of the physical time will increase with increased system size and number of quenches performed, since we determine $t_{\text {MCS }}$ for a given value of $t_{\text {MCBB }}$ by averaging over the corresponding and different values of $t_{\mathrm{MCS}}$ obtained from the different quenches.

This algorithm is purely scalar in nature and very difficult to vectorize or parallelize (apart from simulating different concurrent systems). The overhead structure needs updating only when the configuration changes, therefore the efficiency of the overhead structure increases with decreasing acceptance rate. On a present-day workstation we can perform a quench on a $200 \times 200$ system with $\rho=0.01$ and $T / T_{c}=0.6$ in approximately $1 \mathrm{~h}$ CPU time, when running the quench until $t_{\mathrm{MCS}} \approx 10^{6}$ MCS. This compares favorably with fast algorithms developed for vector computers.

\section{RESULTS}

\section{A. The phase diagram}

The equilibrium phase diagram for the dilute $q=7$ state Potts model in Eq. (2) is shown in Fig. 1. As expected, the diagram consists of three regions: a Potts-ordered phase at low temperatures and low vacancy concentrations, a Potts-disordered phase at high temperatures and/or high vacancy concentrations, as well as a region of phase coexistence. The range of stability of the PO phase is very small (note the logarithmic $\rho$ axis used in Fig. 1).

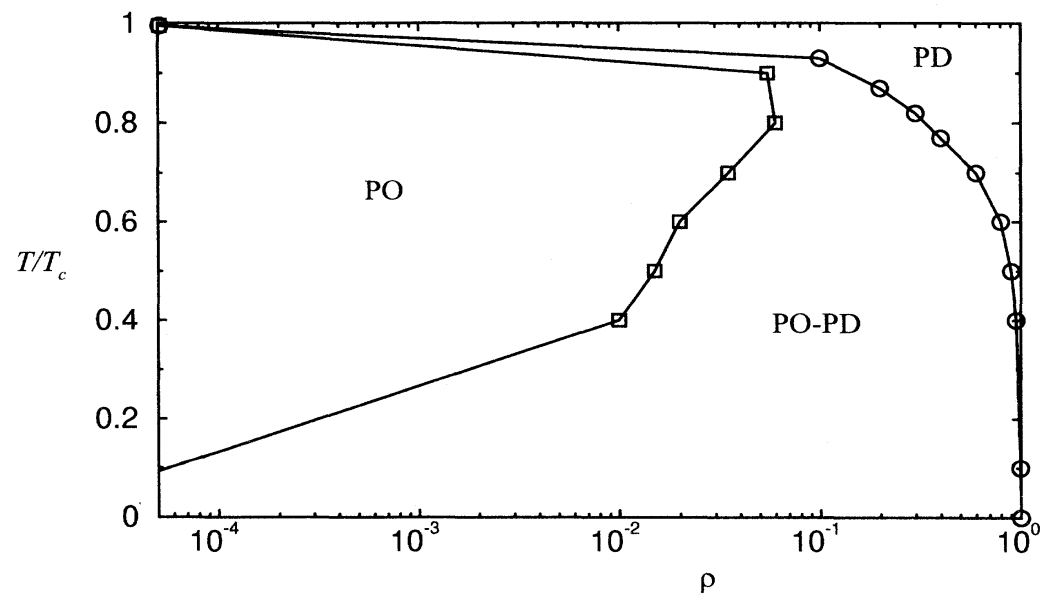

FIG. 1. Phase diagram of the $q=7$ state ferromagnetic Potts model on a triangular lattice with annealed site dilution, cf. Eq. (2). $T_{c}$ is the first-order phase transition temperature of the pure $q=7$ state Potts model. The vacancy concentration is denoted by $\rho$. The digram consists of three regions, the dilute Pottsordered phase (PO), the dilute Pottsdisordered phase (PD), and a region (PO-PD) of phase separation. 

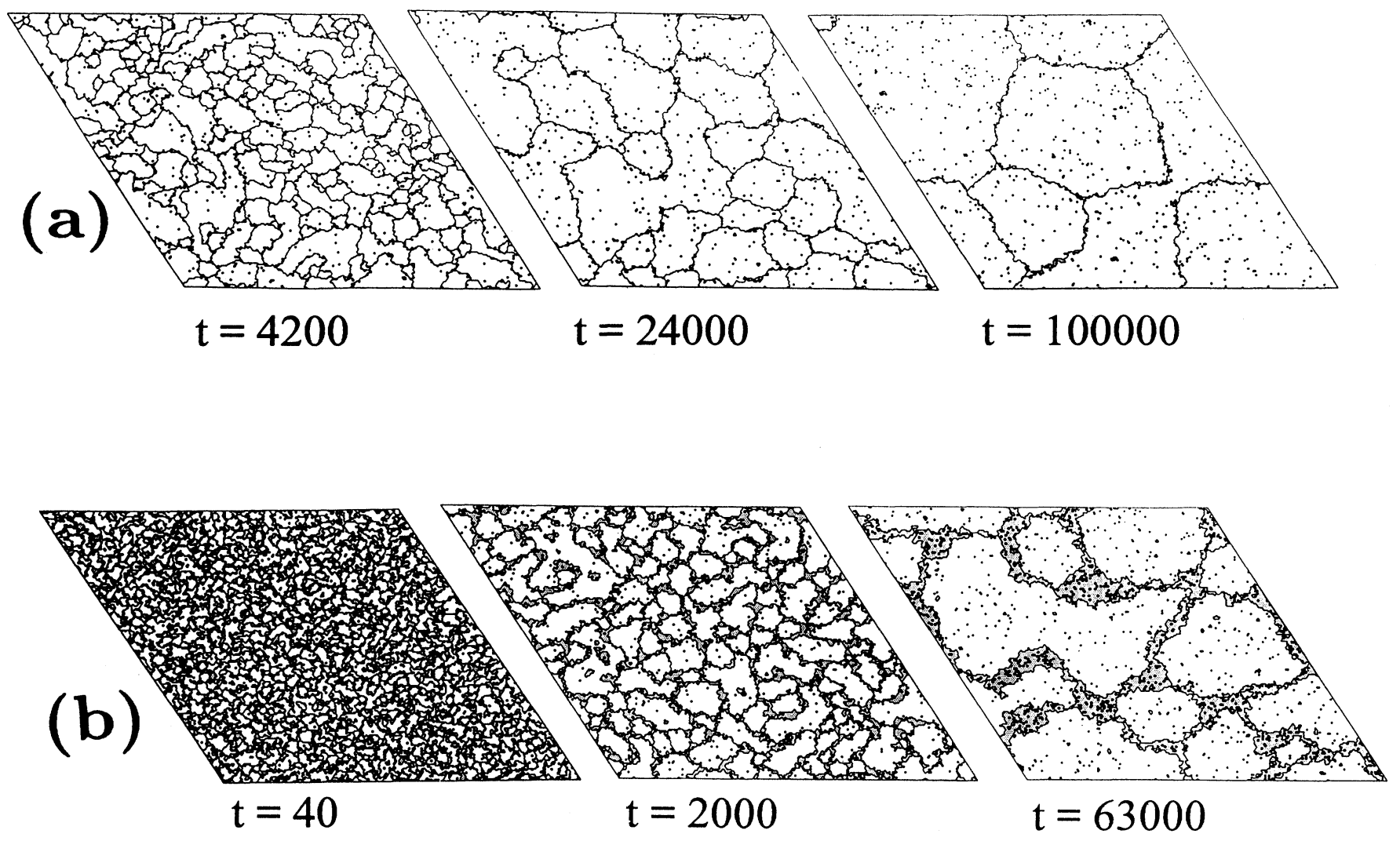

FIG. 2. Time evolution of domain patterns for quenches into the PO phase (a) and into the PO-PD phase-separated region (b) corresponding to vacancy concentrations of $\rho=0.01$ and $\rho=0.2$, respectively. The quench temperature is $T / T_{c}=0.58$. The system sizes correspond to lattices with $200 \times 200$ sites. The domain pattern is mapped out by highlighting the broken Potts bonds. The vacancies are indicated by small dots. The defects seen within the ordered Potts domains are mostly isolated Potts spins or vacancies. The time is in units of MCS.

\section{B. Domain patterns}

Figure 2 illustrates the time evolution of microconfigurations typical for quenches into the PO phase (a) and the PO-PD coexistence region (b), respectively. The microscopic dynamics is the coupled dynamics (A). The configurations in Fig. 2(a) illustrate the coarsening process of the Potts domains as well as the distribution of defects (wrong Potts spins or vacancies) within the domains for quenches into the PO phase. At late times a clear tendency towards accumulation of defects at the domain interfaces is seen. Still, the domain interfaces remain fairly sharp and localized.

The evolution of the domain pattern for quenches into the PO-PD coexistence region appears quite different as seen in Fig. 2(b). First of all, the overall growth rate is much faster as more vacancies are introduced. Second, the PO regions are formed in a compact manner more readily that the $P D$ regions, which seem to nucleate at boundaries between domains with different types of Potts order. In this way the PD phase develops as a connected network of interfacial regions that broaden as time lapses. This interfacial region may be seen as a phase of defects, i.e., a mixture of disordered Potts spins and vacancies.

\section{Growth laws and exponents}

A quantitative analysis of the ordering dynamics in the case of coupled dynamics (A) is provided in Fig. 3, which shows how the length scale $R(t)$ in Eq. (5) evolves in time for quenches into the PO phase, the PO-PD coexistence region, as well for a quench close to the boundary between the PO phase and the PO-PD coexistence region. At late times all three sets of data are seen to display an approximate power-law growth behavior, Eq. (1), with an exponent whose value $n$ seems to be dependent on the vacancy concentration. Even more dependent on the vacancy concentration is the absolute growth rate determined by the amplitude $A$ in Eq. (1). The amplitude strongly increases for increasing vacancy concentration. This is a consequence of the chosen type of dynamics, which obviously is faster the more vacancies there are to facilitate the growth. This behavior is in contrast to that found for the uncoupled dynamics (see also Ref. [17]) where the growth amplitude decreases with increasing vacancy concentration.

A comparison between the growth behavior for the two types of microscopic dynamics, (A) and (B), is provided in Fig. 4, which for a quench into the PO phase at 


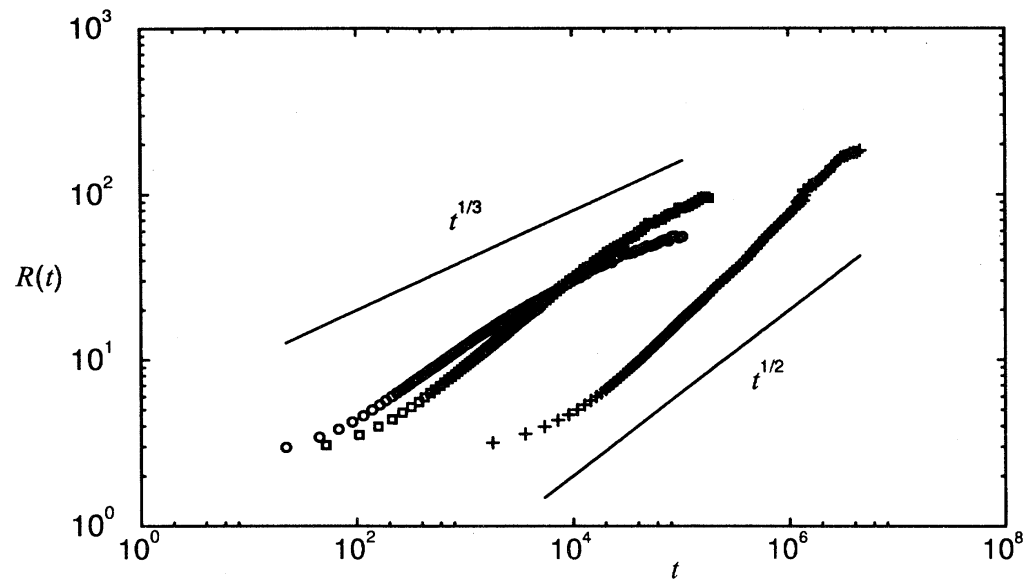

FIG. 3. Double-logarithmic plot of the average size $R(t)$ of ordered Potts domains as a function of time (in units of MCS). The microscopic dynamics is that of coupled vacancy-spin exchange and Glauber spin dynamics. The quench temperature is $T / T_{c}=0.58$. Data for three different vacancy concentrations are shown, $\rho=0.001(+), 0.04$ $(\square), 0.1(O)$, corresponding to quenches into the PO phase, into the crossover region, and into the PO-PD phase-separation region, respectively. The results refer to lattices with $200 \times 200$ sites. Power laws corresponding to $t^{1 / 3}$ and $t^{1 / 2}$ are shown by solid lines. $\rho=0.01$ illustrates that the growth exponent is larger for the coupled dynamics.

A compilation of results for growth exponent values $n$ is given in Fig. 5 for both types of dynamics and for a series of different quench temperatures and vacancy concentrations. When comparing with the phase diagram in Fig. 1 the following tentative conclusion is drawn: In the case of the coupled dynamics the growth exponent pertinent to the PO phase is around $n \simeq 0.6$ whereas in the PO-PD coexistence region $n \simeq 0.3$. In the case of uncoupled dynamics the growth exponent for the PO phase tends towards $n \simeq 0.5$ whereas it is also $n \simeq 0.3$ in the PO-PD coexistence region. We shall in Sec. IV return to a discussion of these results for the growth exponent.

\section{Local order parameters: overshooting effects}

Figure 6 shows the intermediate and late time behavior of the local domain Potts order parameter, $\Psi_{\text {loc }}(t)$ in Eq. (6). The results, which are obtained in the case of coupled dynamics (A), refer to a quench into the PO phase and data for three different system sizes are shown. It is obvious that there is no systematic dependence on the system size in the range shown and that the data therefore represent the thermodynamic limit as far as the system size is concerned.

The most striking feature of the temporal variation of the local domain Potts order parameter is that it is nonmonotonous and displays a maximum. This maximum is above the equilibrium value of the global Potts order parameter, which is indicated by a thin line and an arrow in Fig. 6. Hence at intermediate and late times the Potts order within the domains is larger than the equilibrium order parameter. We shall refer to this effect as an overshooting effect. The overshooting is more pronouced the larger the temperature, as is demonstrated in Fig. 7. This finding suggests that the overshooting may be related to a nonequilibrium entropy effect. We shall return to a discussion of these observations in Sec. IV.

The time evolution of $\Psi_{\text {loc }}(t)$ as a function of vacancy concentration is shown in Fig. 8. Data are here given for a single value of the quench temperature and for various values of the vacancy concentration corresponding to quenches both into the PO phase and into the PO-PD coexistence region. This figure provides two important results. First, there is the overshooting effect, which persists for all vacancy concentrations. Second, the

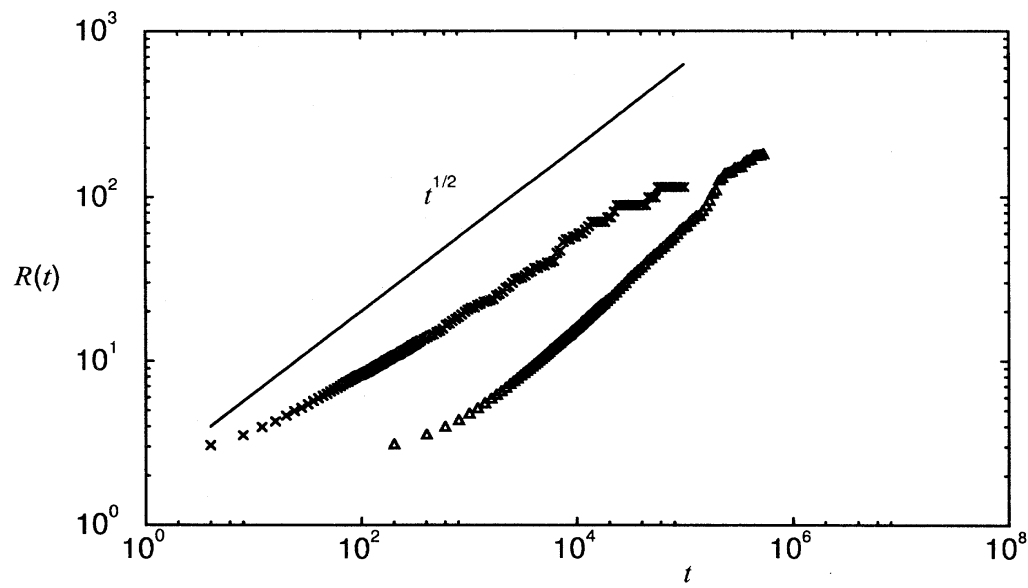

FIG. 4. Double-logarithmic plot of the average size $R(t)$ of ordered Potts domains as a function of time (in units of MCS). Comparison of the growth for two different types of microscopic dynamics: coupled vacancy-spin exchange and Glauber spin dynamics $(\triangle)$ and uncoupled vacancy-spin exchange and Glauber spin dynamics $(X)$. The quenches are in both cases into the PO phase corresponding to a quench temperature of $T / T_{c}=0.58$. The vacancy concentration is $\rho=0.01$. The results refer to lattices with $200 \times 200$ sites. A power law corresponding to $t^{1 / 2}$ is shown by a solid line. 

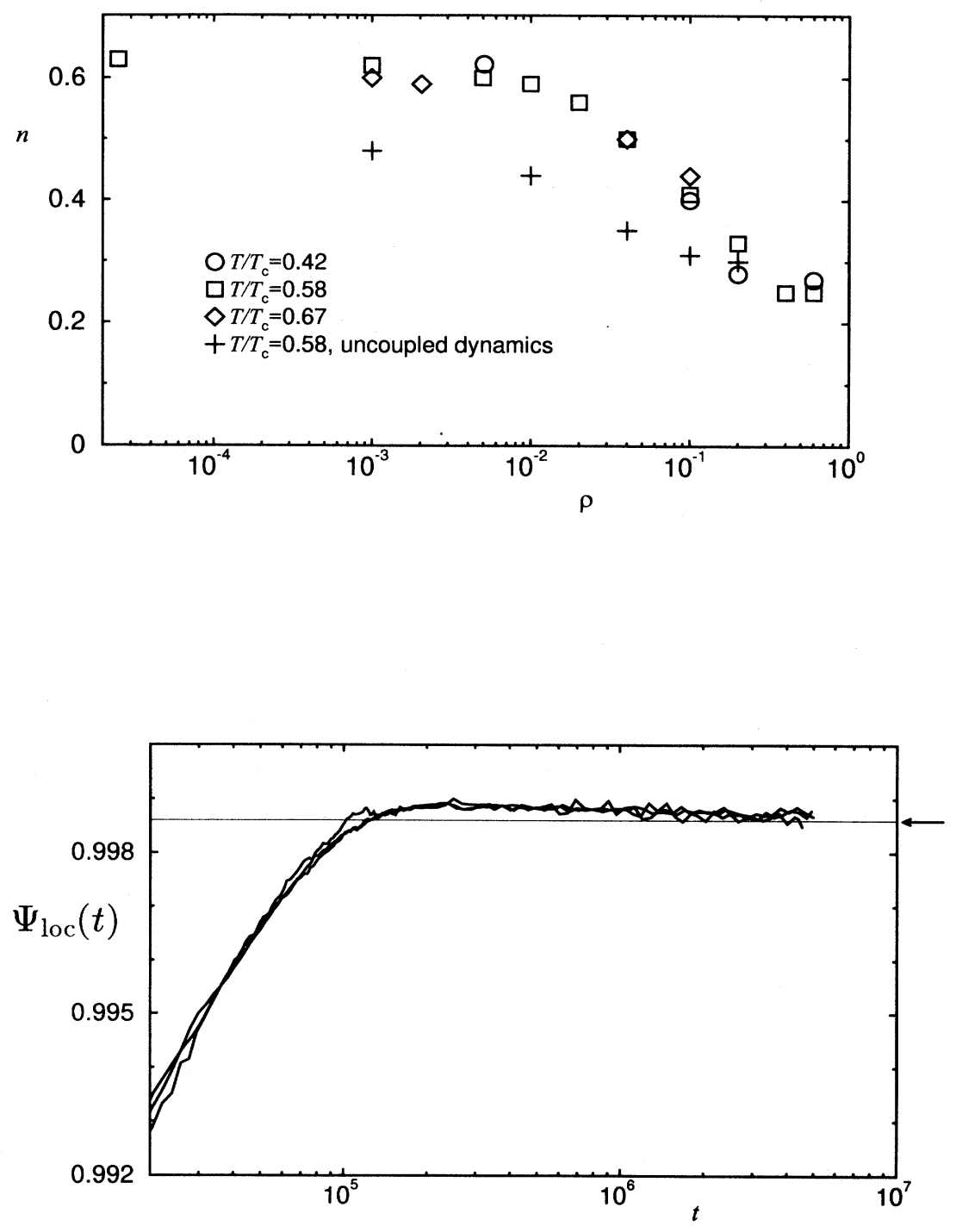

FIG. 5. Growth exponent $n$, cf. Eq. (1), as a function of vacancy concentration shown for quenches to different temperatures. The exponent values are estimated from data for the time dependence of the linear size $R(t)$, as shown in Figs. 3 and 4. Results are presented as obtained for both coupled $(\circ, \square, \diamond)$ and uncoupled $(+)$ dynamics.

FIG. 6. Semilogarithmic plot of the local domain Potts order parameter, $\Psi_{\text {loc }}(t)$ in Eq. (6), as a function of time (in units of MSC). The microscopic dynamics corresponds to coupled vacancy-spin exchange and Glauber spin dynamics. Results are shown for quenches to a temperature $T / T_{c}=0.58$ for a vacancy concentration, $\rho=0.001$, within the PO phase. Data are shown in case of three different lattice sizes, $200 \times 200,500 \times 500$, and $1000 \times 1000$. The equilibrium value, $\Psi_{\text {loc }}(t=\infty)$, of the Potts order parameter is indicted by a thin line and an arrow.

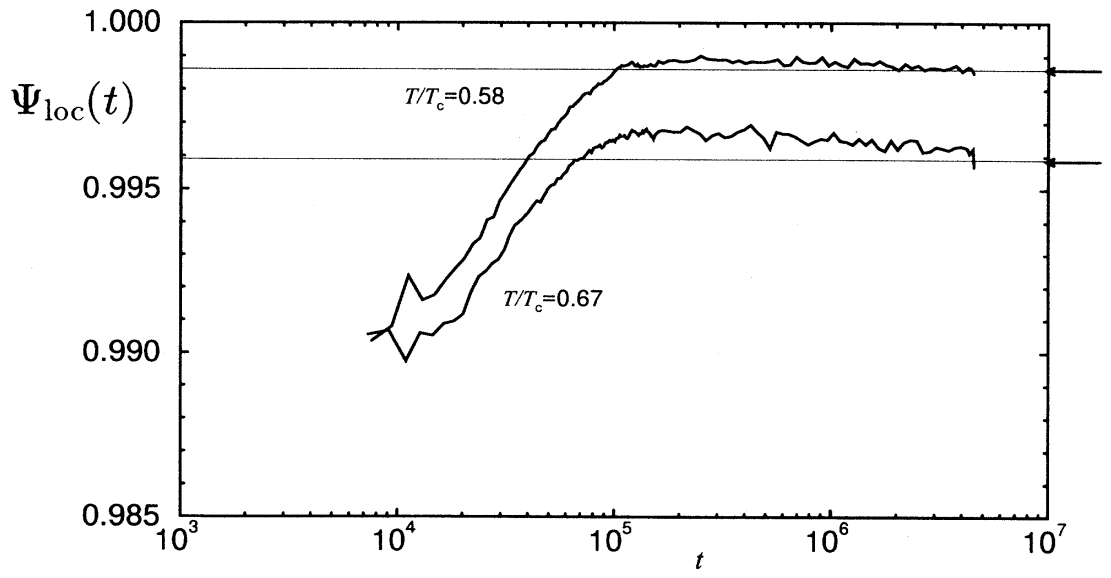

FIG. 7. Semilogarithmic plot of the local domain Potts order parameter, $\Psi_{\text {loc }}(t)$ in Eq. (6), as a function of time (in units of MSC). The microscopic dynamics corresponds to coupled vacancy-spin exchange and Glauber spin dynamics. Results are shown for quenches to two different temperatures, $T / T_{c}=0.58$ and 0.67 , for a vacancy concentrations, $\rho=0.001$, within the PO phase. The equilibrium values, $\Psi_{\text {loc }}(t=\infty)$, of the Potts order parameter in the two cases are indicated by thin lines and arrows. The results refer to a system with $200 \times 200$ sites. 


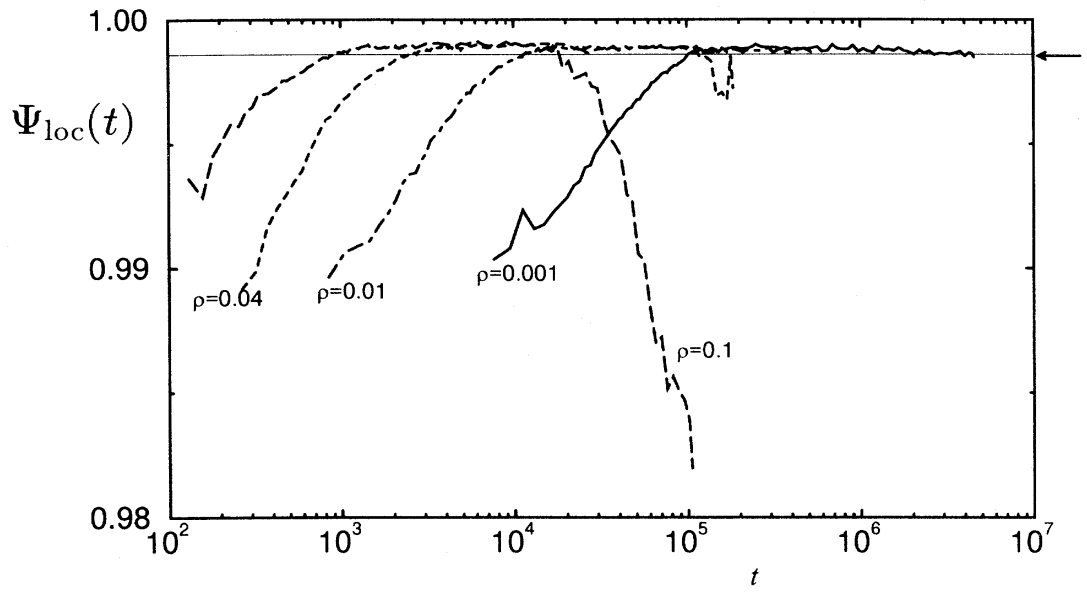

FIG. 8. Semilogarithmic plot of the local domain Potts order parameter, $\Psi_{\mathrm{loc}}(t)$ in Eq. (6), as a function of time (in units of MSC). The microscopic dynamics corresponds to coupled vacancy-spin exchange and Glauber spin dynamics. Results are shown for quenches to a temperature, $T / T_{c}=0.58$, in the case of four different vacancy concentrations, $\rho=0.001$, $0.01,0.04$, and 0.1 . The equilibrium values, $\Psi_{\text {loc }}(t=\infty)$, of the Potts order parameter for $\rho=0.001$ and 0.01 (which are almost the same) are indicated by a thin line and an arrow. The results refer to a system with $200 \times 200$ sites. overshooting is to a level in $\Psi_{\text {loc }}$ that is not sensitive to $\rho$. For quenches into the PO-PD coexistence region, Pottsordered domains are formed transiently with the same high degree of Potts order as seen for quenches into the PO phase. After some time these domains start to disorder again and the corresponding value of $\Psi_{\text {loc }}$ drops to the much lower equilibrium value. Within the time span of the simulation, the equilibrium value of the PO domains in the PO-PD coexistence region has not been reached. For quenches into the coexistence region but close to the PO-PO-PD phase boundary the drop towards the equilibrium Potts order only sets in at very late times. This is seen in Fig. 8 in the case of $\rho=0.04$.

The overshooting effect is not a special feature of the coupled microscopic dynamics. The same effect is found for uncoupled dynamics as demonstrated in Fig. 9. Again the overshooting is to the same level independent of $\rho$ and the drop towards the equilibrium value of $\Psi_{\text {loc }}$ sets in at very late times for quenches into the phase coexistence region.

The overshooting effect is accompanied by a depletion effect in the time evolution of the local vacancy concentration, $\rho_{\text {loc }}$, within the domains. This is shown in Fig. 10, which presents data for $\rho_{\text {loc }}$ for three different values of the global vacancy concentration corresponding to two quenches into the PO phase and a quench into the POPD coexistence region close to the phase boundary. These data sets demonstrate that concomitantly with the overshooting effect in the local Potts order parameter there is a depletion of vacancies within the Potts-ordered domains, for quenches both into the PO phase and into the PO-PD coexistence region. The depletion is seen as a pronounced minimum in $\rho_{\text {loc }}(t)$. This minimum in the local vacancy concentration reflects the fact, observed in the microconfigurations in Fig. 2, that the vacancies are transiently enriched at the domain interfaces.

As described in Sec. II B the simulation of the dynamical evolution proceeds by use of the Metropolis function, $\min \left[1, \exp \left(-\Delta E / k_{B} T\right)\right]$, in the acceptance criterion for the Monte Carlo process. We have verified that the overshooting effects described above are not artifacts of the use of this asymmetric function by repeating some of the simulations with the symmetric Glauber function, $\frac{1}{2}\left[1-\tanh \left(\Delta E / 2 k_{B} T\right)\right]$. It is found that the effects still persist with this function.

In Sec. IV we return to a discussion of these overshooting effects and try to relate them to a generic property of systems far from equilibrium.

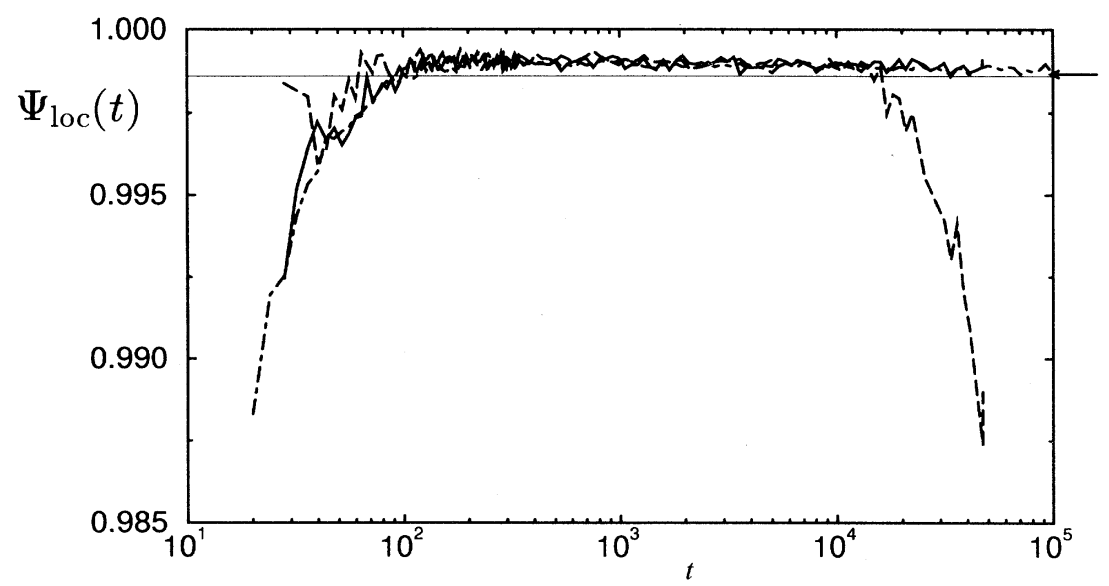

FIG. 9. Semilogarithmic plot of the local domain Potts order parameter, $\Psi_{\mathrm{loc}}(t)$ in Eq. (6), as a function of time (in units of MSC). The microscopic dynamics corresponds to uncoupled vacancy-spin exchange and Glauber spin dynamics. Results are shown for quenches to a temperature, $T / T_{c}=0.58$, in the case of three different vacancy concentrations, $\rho=0.01$ (solid line), 0.04 (dot-dashed line), and 0.1 (dashed line). The equilibrium value, $\Psi_{\text {loc }}(t=\infty)$, of the order parameter for $\rho=0.01$ is indicated by a thin line and an arrow. The results refer to a system with $200 \times 200$ sites. 


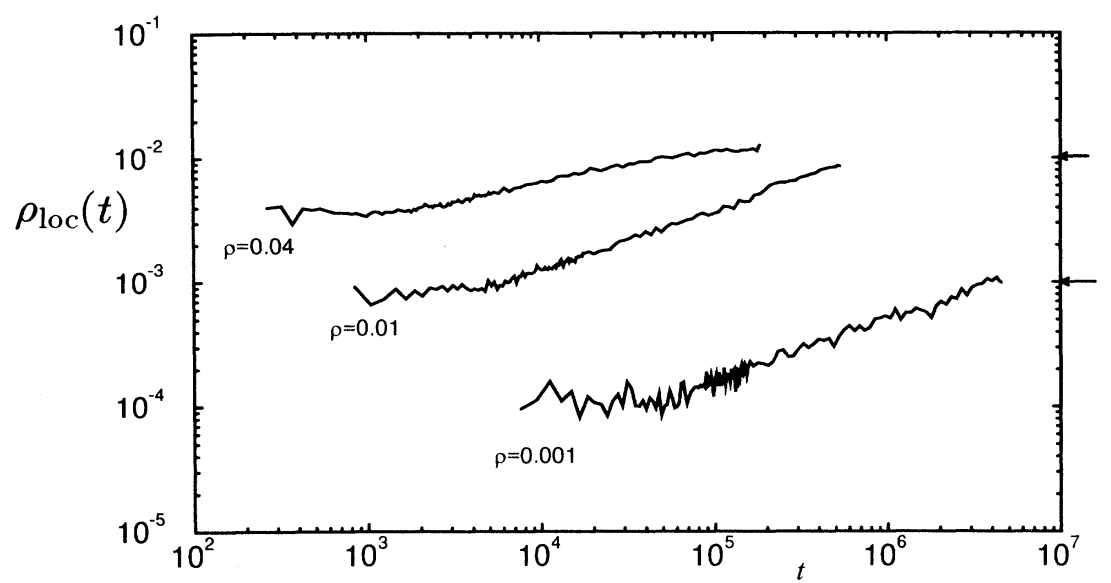

FIG. 10. Semilogarithmic plot of the local domain vacancy order parameter, $\rho_{\text {loc }}(t)$ in Eq. (7), as a function of time (in units of MSC). The microscopic dynamics correspond to coupled vacancy-spin exchange and Glauber spin dynamics. Results are shown for quenches to a temperature, $T / T_{c}=0.58$, in the case of three different vacancy concentrations, $\rho=0.001$, 0.01 , and 0.04 . The equilibrium values, $\rho_{\text {loc }}(t=\infty)$, of the order parameter for $\rho=0.001$ and 0.01 are indicated by arrows. The results refer to a system with $200 \times 200$ sites.

\section{DISCUSSION}

Using a simple model with a first-order phase transition we have in this paper shown by computer simulation that the nonequilibrium ordering dynamics following a thermal quench is very dependent on the degree of annealed site dilution. However, some universality still holds when it comes to the value of the growth exponent $n$ in Eq. (1), that characterizes the late-time algebraic time evolution of the length scale $R(t)$. The data presented in Fig. 5 suggest that $n$ has two possible values, one within the single-phase region (the PO phase) and another one within the two-phase region (the PO-PD coexistence region). Due to crossover effects introduced by finite-system and finite-time limitations inherent in the present numerical approach, there is no sharp transition between the two exponent values at the phase boundary. Intuitively, one may expect that for quenches into this region the system does not "know" to which phase it is quenched until very late times where the role of the mixing entropy, which is a global entity, has fully established itself. It is likely that previously reported anomalously low exponent values in models with diffusing vacancies $[17,19,20]$ suffer from similar crossover effects.

The value of the growth exponent found in the coexistence region, $n \simeq 0$. 3 , is close to the value, $n=1 / 3$, predicted by the classical Lifshitz-Slyozov theory [1] for spinodal decomposition and phase-separation processes. This theory should apply for the present system since the phase separation involves separation of two phases of different density. Hence the growth process is limited by long-range diffusion $[21,53]$. The numerical value of the exponent does not seem to depend on the details of the microscopic dynamics chosen.

The value of the growth exponent found in the Pottsordered one-phase region depends on the type of microscopic dynamics used. In the case of uncoupled dynamics, i.e., a dynamics by which the Potts spins can change value independent of vacancy exchanges, the exponent approaches $n \simeq 0.5$ at low temperatures. This is in accordance with the expectation for a system with a nonconserved order parameter (in this case the global Potts order $\Psi$ ), which should obey the classical Lifshitz-AllenCahn growth law with $n=\frac{1}{2}$ [1]. The presence of an an- nealed dilution should not influence the exponent value but only the amplitude $A$. Only for a tricritical system [53] can the conserved field $\rho$ have an effect on the growth exponent.

In the case of coupled dynamics, i.e., a dynamics by which dynamical evolution in the system proceeds exclusively via hopping of a particle into a vacancy, the numerical exponent assumes a value $n \simeq 0.6$, which is distinctly above the theoretical value. We have found no indications of this being a transient effect although it of course never can be excluded in a numerical study of the present type that a crossover to a different growth law may set in at later times. In a series of studies of the nonconserved Ising model with very low amounts of mobile vacancies (from $\rho=0.0004$ down to a single vacancy), Vives and co-workers [22,24,25] found a growth exponent value of $n \sim 0.6-0.7$ depending on temperature and vacancy concentration. The general trend found was that as the vacancy concentration is decreased the exponent value increases towards 0.7 [25]. These results are in close agreement with our findings for the dilute Potts model with a dynamics controlled by vacancy hopping. Furthermore, the decrease in the exponent value as the temperature is increased as suggested by the data in Fig. 5 is also consistent with the results for the nonconserved Ising model [25]. It has been suggested by Frontera, Vives, and Planes [25] that the anomalously high growth exponent found for low vacancy concentrations and low temperatures in the nonconserved Ising model with vacancy hopping is caused by the strong localization of non-correlated vacancies in the domain boundaries. Whereas a vacancy diffusing within an ordered domain undergoes a normal random walk, a vacancy trapped in the domain boundary undergoes some kind of selfavoiding random walk associated with an anomalous diffusion constant corresponding to diffusion in a constrained geometry [25].

Returning to the results for the growth exponent in the case of uncoupled dynamics, the numerical value of the exponent at finite temperatures is found to be slightly below the theoretical value, which is likely to be a finitesystem and finite-time effect. This is consistent with previous findings for pure $q$-state nonconserved Potts models with large $q$ where the asymptotic region is only reached 
for very large systems, $N \sim 1000^{2}$, and at very late times [54].

Vacancy hopping as the main mechanism for phase separation and spinodal decomposition has previously been studied by Monte Carlo simulation for symmetric and asymmetric $A B$ alloys modeled by the conserved Ising model with small amounts of vacancies. Specifically it was shown by Yaldram and Binder [27-29] that a few percent of mobile vacancies produced the same results for the ordering dynamics as the standard Kawasaki $A-B$ exchange mechanism in pure systems. However, the asymptotic region characterized by the exponent value $n=\frac{1}{3}$ was not reached in these studies but rather the well-known transient region characterized by an effective exponent value of $n \simeq 0.25$ [52]. Subsequently it was found by Fratzl and Penrose [26] that an exponent value close to $\frac{1}{3}$ could be obtained in the conserved Ising model with vacancy diffusion if the vacancy concentration was substantially reduced to $\rho=6 \times 10^{-5}$. In the light of the findings of an anomalously high growth exponent by Vives and co-workers [24,25] for the nonconserved Ising model with vacancy hopping it is possible, however, that the exponent value reported by Fratzl and Penrose [26] may be influenced by two counteracting effects: one being the usual slow transient involved in spinodal decomposition and the other being a fast mode controlled by the localized vacancy diffusion in the domain boundaries.

Finally, we discuss the overshooting effects found in the local domain order parameter and the concomitant depletion effect in the local domain vacancy concentration, cf. Figs. 6-10. It is likely that these effects are due to some generic mechanism in far-from-equilibrium ordering processes. First, we have found in the present paper that the effects are robust to changes in the microscopic dynamics as well as to changes in composition and temperature. Second, it was shown in a recent study of the conserved $q=2$ (Ising) and $q=3$ state twodimensional Potts models [52] that the local concentrations of Potts excitations (which are the carriers of growth within the condensation-evaporation phenomenology) within the ordered Potts domains display a minimum as a function of time. This is equivalent to a transient depletion of disorder, and hence an overshooting in the domain Potts order parameter. In that study [52] it was suggested that the overshooting effect may explain the slow transient growth mode in spinodal decomposition characterized by $n \simeq 0.25$. Finally, we have recently shown [55] using the simplest possible model of an order-disorder process, the two-dimensional nonconserved Ising model, that overshooting is very pronounced in the local domain order of this model as well, and in the case of site dilution there is a concomitant depletion of vacancies within the ordered Ising domains.

An important observation is the temperature dependence of the overshooting effect as demonstrated in Fig. 7 which shows that the degree of overshooting is larger for the higher temperature. This suggests that the effect is related to entropy. Furthermore, it is striking that the level of the transient local order parameter, cf. Figs. 8 and 9 , does not seem to be very dependent on the vacancy concentration or the choice of microscopic dynamics. These observations suggest that in the transient state, vacancies, and spin excitations are expelled from the growing ordered domains, which hereby increase the internalenergy contribution to the lowering of the nonequilibrium free energy. The disorder (and hence the entropy) is localized in the domain boundaries and in the global domain-boundary configuration. Only at later times does the stabilizing effect of the mixing entropy manifest itself. This leads to a redistribution of the vacancies which start dissolving in the ordered domains together with a decrease of the domain Potts order towards the thermodynamic equilibrium value.

Obviously, during the quench the total entropy of the system decreases in monotonous fashion and so does the total internal energy. However, the energy is a local quantity and it can be easily minimized by local rearrangements involving spin ordering and exclusion of vacancies from ordered domains. A balancing contribution in the free energy to this ordering effect and minimization of internal energy is the entropy of mixing, which, however, is a global property of the system that only manifests itself fully over a much longer time scale. This subtle competition between nonequilibrium energy and entropy is what we believe is the mechanism behind the overshooting effects.

We are only aware of a single piece of experimental work on ordering dynamics that provides the kind of time-resolved early-time structural information that can be used to address the question of the possible presence of overshooting effects. Park et al. [56] report from an $\mathrm{x}$ ray scattering study of the ordering process in $\mathrm{Fe}_{3} \mathrm{Al}$ that the short-range order goes through a transient maximum in contrast to the long-range order parameter, which monotonically approaches the equilibrium value. These observations were interpreted as a consequence of a subtle coupling between long- and short-range order characterized, respectively, by the correlation length of the order-parameter fluctuations and the domain size [56-58]. We suggest that the observations made by Park et al. may be an example of the general phenomena we have described in the present paper. It would be obviously of interest to further analyze the early-time dynamical structure factor obtained from time-resolved scattering experiments on various systems with an aim to scrutinize the occurrence of possible overshooting effects in farfrom-equilibrium ordering processes.

\section{ACKNOWLEDGMENTS}

This work was supported by the Danish Natural Science Research Council under Grant No. 11-0065-1 and partially supported by the MRL Program of the National Science Research Foundation under Grant No. DMR9123048. Comments on our work by Hans C. Fogedby are gratefully acknowledged. 
[1] J. D. Gunton, M. San Miguel, and P. S. Sahni, in Phase Transitions and Critical Phenomena, edited by C. Domb and J. L. Lebowitz (Academic, New York, 1983), Vol. 8, p. 267.

[2] H. Furukawa, Adv. Phys. 34, 703 (1985).

[3] O. G. Mouritsen, in Kinetics of Ordering and Growth at Surfaces, edited by M. G. Lagally (Plenum, New York, 1990), p. 1.

[4] For an experimental overview of the field of ordering dynamics, see the selection of papers in Dynamics of Ordering Processes in Condensed Matter, edited by S. Komura and H. Furukawa (Plenum, New York, 1988).

[5] A. J. Bray, Adv. Phys. (to be published).

[6] O. G. Mouritsen, P. J. Shah, J. V. Andersen, H. F. Poulsen, and H. Bohr, Phys. Scr. T38, 55 (1991).

[7] O. G. Mouritsen and P. J. Shan, in Kinetics of Ordering and Growth at Surfaces (Ref. [3]), p. 45.

[8] J.-K. Zuo, G.-C. Wang, and T.-M. Lu, Phys. Rev. Lett. 60, 1053 (1988).

[9] D. J. Srolovitz, M. P. Anderson, G. S. Grest, and P. S. Sahni, Acta. Metall. 32, 1429 (1984).

[10] G. S. Grest and D. J. Srolovitz, Phys. Rev. B 32, 3014 (1985).

[11] D. J. Srolovitz and G. S. Grest, Phys. Rev. B 32, 3021 (1985).

[12] D. A. Huse and C. L. Henley, Phys. Rev. Lett. 54, 2708 (1985).

[13] W. Schreier, G. Besold, and K. Heinz, Vacuum 41, 412 (1990).

[14] D. Chowdhury, M. Grant, and J. D. Gunton, Phys. Rev. B 35, 6792 (1987).

[15] O. G. Mouritsen, Phys. Rev. B 32, 1632 (1985).

[16] G. F. Mazenko and O. T. Valls, Phys. Rev. B 33, 1823 (1986).

[17] D. J. Srolovitz and G. N. Hassold, Phys. Rev. B 35, 6902 (1987).

[18] T. Ohta, K. Kawasaki, A. Sato, and Y. Enomoto, Phys. Lett. A 126, 93 (1987).

[19] O. G. Mouritsen and P. J. Shah, Phys. Rev. B 40, 11445 (1989).

[20] P. J. Shah and O. G. Mouritsen, Phys. Rev. B 41, 7003 (1990).

[21] O. G. Mouritsen, P. J. Shah, and J. Vitting Andersen, Phys. Rev. B 42, 4506 (1990).

[22] E. Vives and A. Planes, Phys. Rev. Lett. 68, 812 (1992).

[23] J.-F. Willart, O. G. Mouritsen, J. Naudts, and M. Descamps, Phys. Rev. B 46, 8089 (1992).

[24] E. Vives and A. Planes, Phys. Rev. B 47, 2557 (1993).

[25] C. Frontera, E. Vives, and A. Planes, Phys. Rev. B 48, 9321 (1993).

[26] P. Fratzl and O. Penrose, Phys. Rev. B 50, 3477 (1994).

[27] K. Yaldram and K. Binder, Z. Phys. B 82, 405 (1991).
[28] K. Yaldram and K. Binder, J. Stat. Phys. 62, 161 (1991).

[29] K. Yaldram and K. Binder, Acta. Metall. Mater. 39, 707 (1991).

[30] J. H. Oh and D.-I. Choi, Phys. Rev. B 33, 3448 (1986).

[31] A. E. Jacobs and C. M. Coram, Phys. Rev. B 36, 3844 (1987).

[32] D. P. Belanger, A. R. King, and V. Jaccarino, Phys. Rev. B 31, 4538 (1985).

[33] H. Yoshizawa, R. A. Cowley, G. Shirane, and R. Birgeneau, Phys. Rev. B 31, 4548 (1985).

[34] M. Grant and J. D. Gunton, Phys. Rev. B 29, 1521 (1984).

[35] J. Villain, Phys. Rev. Lett. 52, 1543 (1984).

[36] G. Grinstein and J. F. Fernandez, Phys. Rev. B 29, 6389 (1984).

[37] E. T. Gawlinski, K. Kaski, M. Grant, and J. D. Gunton, Phys. Rev. Lett. 53, 2266 (1984).

[38] E. T. Gawlinski, S. Kumar, M. Grant, J. D. Gunton, and K. Kaski, Phys. Rev. B 32, 1575 (1985).

[39] S. R. Anderson and G. F. Mazenko, Phys. Rev. B 33, 2007 (1986).

[40] D. Chowdhury and J. D. Gunton, J. Phys. A 19, L1105 (1986).

[41] M. Grant and J. D. Gunton, Phys. Rev. B 35, 4922 (1987).

[42] D. A. Huse, Phys. Rev. B 36, 5383 (1987).

[43] S. R. Anderson, Phys. Rev. B 36, 8435 (1987).

[44] M. Hillert, Acta Metall. 36, 3177 (1988).

[45] H. Gilhøj, M. Laradji, B. Dammann, C. Jeppesen, O. G. Mouritsen, S. Toxvaerd, and M. J. Zuckermann, Math. Comp. Sim. (to be published).

[46] M. Laradji, H. Guo, M. Grant, and M. J. Zuckermann, Adv. Chem. Phys. 89, 159 (1994).

[47] T. Kawakatsu, K. Kawasaki, M. Furusaka, H. Okabayashi, and T. Kanaya, J. Chem. Phys. 99, 8200 (1993).

[48] J. H. Yao and M. Laradji, Phys. Rev. E 47, 2695 (1993).

[49] M. Laradji, O. G. Mouritsen, S. Toxvaerd, and M. J. Zuckermann, Phys. Rev. E 50, 1243 (1994).

[50] F. Y. Wu, Rev. Mod. Phys. 54, 2235 (1982).

[51] A. Milchev, K. Binder, and D. W. Heermann, Z. Phys. B 63, 521 (1986).

[52] C. Jeppesen and O. G. Mouritsen, Phys. Rev. B 47, 14724 (1993).

[53] W. W. Mullins and J. Viñals, Acta Metall. 37, 991 (1989).

[54] G. S. Grest, M. P. Anderson, and D. J. Srolovitz, Phys. Rev. B 38, 4752 (1988).

[55] H.Gilhøj, C. Jeppesen, and O. G. Mouritsen (unpublished).

[56] B. Park, G. B. Stephenson, S. M. Allen, and K. F. Ludwig, Phys. Rev. Lett. 68, 1742 (1992).

[57] G. F. Mazenko, O. T. Valls, and M. Zannetti, Phys. Rev. B 38, 520 (1988).

[58] B. Morin, K. R. Elder, and M. Grant, Phys. Rev. B 47, 2487 (1993). 